\title{
Developing an Open Book Examination in a Sensor Technology Course
}

\author{
A. J. Rissanen, Member, IACSIT
}

\begin{abstract}
According to the Bologna declaration, the Finnish National Defence University (NDU) provides undergraduateand graduate-level courses in academic and military disciplines. Military technology is one of the major learning aims at the NDU. Learning aims in master level technology courses are on scientific knowledge, understanding how technology is related to science, and life-long learning in technology-based working environments. Sensor technology is one of the key elements for surveillance and target acquisition principles. This study presents one development concept in assessment research and how it has been applied to learning sensors. Obligatory lectures and exercises are specific to NDU's teaching environment. As such, they are effective methods for co-operative learning and teacher resource utilization. On the other hand, distance education features have been actively added to NDU's curriculum structures. Exams can estimate students' learning outcome and act as tools to diversify teaching processes. When aiming for better student motivation and learning results, enough space must be given for students' contributions in examination protocols.
\end{abstract}

Index Terms-Action research, distance education, open book examination.

\section{INTRODUCTION}

The modern university is continuously challenged to do things better. One of the key issues is to improve general efficiency by looking at applied processes and resource utilization. New technologies open up opportunities for new ways to achieve education aims. Demands to improve people's abilities to learn scientific knowledge, obtain new information, and resolve real-world problems are widely expressed and need to be included in existing pedagogical manuscripts and future curricula. Technical innovations have changed the teaching and learning practice. On the other hand, when looking at how examinations are held, there are many practical reasons for using paper and pen types of exams with only slight modifications. These classical examinations are constructed in a way that students must answer based on what they can memorize or guess the right answer. It is also true that this type of examination is often straightforward in terms of formulating with a grading schema based on counting the right answers or asking for a list of details. Moreover, everybody is at the same starting point; pondering issues, such as technology-based superiority or utilization of note taking, is needless.

Compared to classroom course tests, examinations proofing work-life-oriented practical skills may often include

Manuscript received July 24, 2014; revised September 27, 2014.

A. J. Rissanen is with the National Defence University, Santahamina, 00861 Helsinki, Finland (e-mail: antti.rissanen@mil.fi). parts where the examinee must first obtain correct, up-to-date information on the necessary procedures before taking any action. As a result, multiple items are needed when instructors estimate each student's ability to solve given tasks with supplementary information. Even in many academic professions, the need for skill-based evaluations has been noticed. For academic courses, the essential skills are general and contexts sensitive. This complexity is the main reason why it is difficult to form an adequate mix in the exam. In addition, when considering lifelong learning, it is not enough for students to obtain current professional knowledge because in the future they must be skillful workers with the ability to apply their knowledge and skills in new situations.

At the NDU, students must learn a comprehensive set of concepts covering many areas in science related disciplines. The target in all applied science courses and especially in master degree studies is that the officer understands the phenomena behind the application, knows the basic technology principles, and is able to gain more knowledge on the topics. As a result, each branch of the curriculum needs to be formulated as a general guideline for learning aims and methods around which group profile adjustments can be done. Furthermore, to be able to label and point skills in multiple learning aims the master program at the NDU has relatively short courses. This means that exams are an essential part of each theme's learning. The aim of this study is to describe how long-term examination development in one specific course is done. Changes in learning outcome, learning environment, and students IT skills have been followed in the sense of action research principles.

\section{A. Action Research Methodology}

Action research supports educators in seeking out ways in which they can provide enhanced quality; therefore, it is worthy of consideration [1]. Action research is designed to bridge the gap between research and practice. Kemmis and McTaggart [2] propose that action research forms a spiral of process stages. However, they emphasize that in reality the process may not be as neat as the spiral of self-contained cycles of planning, acting, observing, and reflecting suggests. These stages may overlap and initial plans may become obsolete based on acquired new data from the experience. This makes the process also more fluid, open, and responsive. In O'Leary's model [3], action research is considered an experiential learning approach, where aims include continuous needs to refine the methods, data, and interpretation based on gained understanding from earlier cycles.

Action research methodology describes a practical approach to solving a detected learning environment problem. In this study, the examination is considered as a leading issue 
for better learning outcomes. Some of the changes in the environment and course structure are important aspects of the study that need explanation.

\section{EXAMINATION METHODS}

\section{A. Aims of Exam in Military Environment}

Some courses in Finnish officers' education have a wide set of similarities with courses held in other universities. Therefore, in disciplines where universal skills that are not too deeply bonded to any specific profession, planning examinations may utilize traditions or the best practices common to universal learning aims. In military professions, assessment may mean examination, trial, proof, or a series of questions or problems designed to determine knowledge or competency. The primary goal is to determine if training does what it is designed to do with attention on efficiency and affectivity. In this area, the purpose of an exam is to measure skills, knowledge, intelligence, abilities, or other aptitudes of an individual (or group). Even in the academic disciplines of military education, tests are designed by keeping in mind the terminal learning objective. The terminal learning objective is what a soldier or officer must be able to perform in peace and war. On a detailed level, this is sometimes very difficult because NDU trains officers from the bachelor level up to senior officer level in an officer's career. As a result, terminal professional objectives for the best of the students are at the level where the main processes of the Finish defense forces are planned and lead.

\section{B. Closed Book Testing Method}

Traditional academic examinations have been conducted using the so-called closed book method. These examinations are considered formal, end-of-semester, timed examination in which students fill forms, make calculations, or write essays. Personnel's role is to give out questions or answer forms, prevent communication between students, and ensure nobody uses any notes. At the end of the examination, papers are collected. In a semi-computerized examination, student response material may be printed out or electrically stored for scoring.

The educational model in the closed book examination assumes that students answer questions on the strength of their intelligence and ability to recall information. In principle, any other information except that provided by the examiner for the purposes of the test cannot be used. In practice, the method is not so pure. In some disciplines, drawing equipment, calculators, equation lists, or answer books are utilized. The length of closed book exam is typically limited to a few hours.

In university learning environments, paper-based closed book examinations dominate. The benefit of conducting traditional exams is that they are considered cost- effective, and they give a fair assessment [4]. Even if the need for modernization is pondered, the practical reason to select the old system is because any extra investments on examination technology can be postponed for a few years. For instructor practice, most closed book test questions or forms are reusable if the course's textbook or lecturing set has not changed extensively or the test instrument itself has not turned out to be defective.

Examination protocols that follow the closed book method have been widely criticized as 1) being a poor test tool, 2) not revealing variations in understanding, 3) not supporting learning and finally, 4) not reflecting real world situations (e.g., [5]). Another challenge facing the closed book method is failure rates (relative high failure rates in first year's exams and subsequently high course failure rates, e.g., [6]). Sometimes, teachers may even think that they are teaching for the test or at least spending most of the lecturing time preparing students for the test.

One of the aims in university level teaching is to help students to think critically, promote skills to analyze situations, and find possible solutions. The leading aim in all levels of NDU's science and engineering education for officers is to enhance their understanding of the essence of phenomena and show the ways in which science has been utilized in military technology. However, when the purpose is to measure terminological issues, general knowledge, or selected calculation skills, then the closed book methodology moderately serves examination needs for military technology-related courses.

\section{General Open Book Testing Method}

One of the formal definitions of generic open book testing states that it is a written examination during which an examinee is permitted to consult references to answer questions calling for organization, analysis, or judgment, rather than memorization [6]. For practical reasons, the amount of material to be utilized can be limited. Reasons for limiting of reference material are to 1) limit choices in answers, 2) consider of allocated time for examination, 3) focus and alignment in the assessment, or 4) have desk space to store the material at the examination premises. A properly constructed open book exam requires students to use the book and apply that knowledge to formulate successful answers. Any issues on cheating (test security) are considered minimal. On the other hand, preliminary actions are necessary, such as actively encouraging students to study additional sources of knowledge itself with an idea of what is available and where.

One of the goals in open book assessment is to find where student are in their learning process. A teacher should prepare students for the test environment in which they are going to be tested. Open book testing stresses the importance of retrieval skills and students' abilities to find and access information. Less value is given to the importance of memorizing facts that the discipline and course itself may include as general knowledge for professionals. These type constrains should be clarified before the students are guided to study the course content and prepared for the final examination. From a student perspective, an open book test requires the use of critical thinking skills to pass successfully. Management of the lectured information is linked not only to a specific textbook or compendium but to electrically available information.

Computerized learning material and learning events have become common due to common learning management system (LMS) utilization in higher educations. Both 
educators and researchers are eager to develop skills that guarantee how computerized learning and testing may be utilized for better outcomes. As a result, the web has opened new ways to make the open book test to work even in an online environment.

\section{Computerized and Advanced Open Book Testing Methods}

Digitalized university concept has been widely studied and is already the corner stone in many faculties. On the other hand, many of the promised advantages need to be implemented on a per discipline basis or even rearranged for global thinking. Computerized, locally managed, and teacher-made tests became common due to the wide use of computers on campus and the appearance of learning management systems (LMS) [7]. At the NDU, moving from the proprietary LMS e.g., to Moodle, has established deeper interest in long-term development for enhanced assessment methods.

Instructors have three basic options for delivering computerized testing: 1) each student takes the closed book exam in a specific computerized classroom with the instructor or other personnel taking the same role as in the closed book exam; 2) as above, but it is a computerized open book exam in a classroom, or 3) the instructor delivers the digital exam material as a take-home examination. Generally, the first two options mean that every student brings their own laptop to the classroom or the exam has to be administered in a specific class with the necessary equipment. In the presence of a proper online learning management system, the teacher may choose a take-home exam method. However, necessary environmental parameters must be clarified for students.

The case material in this study is constructed around the question of how to extend a generic open book method to the direction of the computerized take-home exams. Only qualitative data and face-to-face type opinions were collected and analyzed.

Computerized testing and automatic grading with modern learning management system has raised lot of interest. However, the grading of written answers often needs contribution from the teaching staff, but this part of the examination is often most fruitful for analyzing gained knowledge or informal learning aims, including attitudes. At the NDU, some of the exercises are automated in the Moodle or assigned remotely with a specific mathematical test bench. For all examinations so far, instructors have used personal observation in grading.

Despite the digitalized learning environment offering many advantages to be utilized, there have been and still are serious concerns associated with computerized testing. Until recently, internet-based testing has had both hardware and software type concerns for robust functionality. Lately, themes like cheating or general security of the test content have raised concerns.

\section{CASE}

\section{A. Remarks of the Sensor Course}

At the NDU, principles of surveillance and target acquisition are discussed from multiple viewpoints and in many course levels. This study makes observations on the first pre-Bologna declaration master level course on sensor technology (2005) up to the fourth revised Bologna declaration type course (spring 2014). The upper learning objectives of the course are the skill to see limits and opportunities for applied technologies, skills to utilize physics concepts, and logical formulation of the presented phenomena.

According to the Bologna declaration's principles, qualified education means that customer and process quality challenges must be broadly considered. A valid assessment is fit for purpose and measures tasks which it is supposed to measure [8]. Fair assessment utilizes many different measures and emphasizes that students be encouraged in this process [9]. In an instructional evaluation, diagnostic evaluation declares the current status, formative evaluation supports planning and instructional activities, and a summative part enables decision making and conclusions [10].

\section{B. First Phase: Open Book Examination}

The sample in the first phase consisted of five successive courses between 2005-2009 with 57 students majoring in technology studies. Lectures and exercises were held in a phase system over six weeks. Each week was filled with multiple obligatory activities for an average of seven hours per day, five days per week. The sensor technology course was held from the viewpoint of surveillance and target acquisition in 90 planned studying hours. Most of the time was spent in teacher-lead education, involving providing information, explaining formulation for calculations, and utilization of some spreadsheet tools. At the end of the course, each student team (2-3 persons) had to present a 10 minute introduction on a selected theme. Generally, the learning technique was good because the one-year master stage was followed immediately after the three-year bachelor stage. The knowledge level of the students was up-to-date because previously learned concepts were still in mind. Even earlier learned physics concepts were utilized in presentations. Instead of repeating theory from science courses, the exam aimed to be more adaptive.

In the examination, the questions were constructed on tasks: explain concepts, calculate signal parameters, and make predictions according to calculation and learned principles. The test consisted of five main question areas (five main questions with connected sub-questions). To make assessment fair and practical, all tools, including electric and printed version of the textbook and other lecturing material, were available in the examinations. Due to a three-hour time limit in the examination, good preparation was essential task for good grades. Some examples of questions and models show how to answer and ways of refining information in texts e.g., concept maps were presented during lectures. The majority of the students were spending over 100 minutes writing answers and some wrote to the very last minute. Nobody failed. However, the answer sample varied from general to more detailed answers. Based on the formal and informal feedback, it was obvious that the amount of effort both in examination and during the whole course had obvious links to personal grades. 


\section{Second Phase: Open Book-Home Examination}

The sample in the second phase consisted of four successive master courses between 2009-2014. The learning environment was based on NDU's Bologna declaration curriculum plan. All of the 53 students had served more than four years in the officer's profession after their bachelors in military studies. As a new dimension for the surveillance and target acquisition approach, some of the students were professionally trained in some of the areas of application. The course was an eight-week lecture and study period at the beginning of the second semester. The early stage of the six-semester master's degree program was a good time to undertake many unobvious learning procedures.

\section{Environment in the "Take Home Examination"}

Almost at the same time as starting the Bologna declaration compatible master program, the learning management system in question was transferred to the Moodle environment. Based on earlier experimentation and robustness of the LMS, blended distance education was applied to all academic and selective profession-oriented sections. During the general semester, students received three days of lecturing and guided exercises each week (obligatory presence) from a set of disciplines. The remaining two days were managed remotely by portal supported self-study.

In the sensor course, the purpose was that students could 1) clearly identify the problem, 2) find alternatives, 3) plan changes, 4) implement changes, and 5) engage in follow up.

Examination questions and selected course material was given in the portal. Answers were uploaded and stored in the LMS. Questions were given out the previous day after 5:00 PM and students uploaded their answers to the portal, which was closed the following examination day at 1:00 PM. Some of the students took the examination in the class but most of them were remote. Minor adjustments were made on the rules of how the fully portal-based exam was held.

Exams were constructed so that they required students to use one source and apply that knowledge to find supporting information and formulate deeper and successful answers. Structure of the Observed Learning Outcome taxonomy (SOLO) describes systematically how a learner's performance grows in mastering knowledge. Therefore, it is important to formulate questions so that they do not inherently limit chances to achieve highest taxonomical levels in responses. [11] Questions contain guidelines, which are meant to help students to construct thoughtfully planned answer structures. Moreover, explicit maximum credit points in each section may help students to predict what is considered the right level and length of a model answer.

Unnecessarily long and overly detailed answers in basic concepts take too much time and detract from pondering more professional sections of the exam. Mainly based on feedback, the amount of longer essay-type questions was lowered and replaced with relatively simply defined concepts to start with. In addition, to get fair grading, the effort level and time consumption was settled to certain limit. Because all questions were not set to invite responses at higher SOLO levels, the idea of having the only "big question" made a major difference for those who aimed for top grades.

\section{E. Student Activation by "Take Home Exam"}

Studies on exercise material often show that increased use of exercises or demonstrations may improve students' understanding of new phenomena. Open book tests bring more options to formal examination protocols, which are motivators. This also gives less motivated students a new way to show their competence and skills. All students tried to survive the given tasks in the open book tests. Hence, the open book test as an option could be a positive alternative when the purpose is to engage students in demanding areas.

In addition, students' theoretical understanding may be improved when the student tries to clarify the meaning of a certain phenomenon by an open book test. In general, the purpose is to offer instruction and courses that could attract interest and creativity when theoretical tasks are in question. However, the more challenging task is to offer examinations that also could provoke students' interest.

Lecturers use a lot of time in creating inspiring lecture experiences but use less time in pondering how to change the test procedures or how to generate new models. This is understandable when the purpose is to have justified scoring of a course when time resources are tight. However, a wider selection of testing protocols may also produce comprehensive skills and improve theoretical and conceptual understanding. The open book examination, with its modified take-home versions, offer only one option for differentiating formal examination protocols. The meaning of examination is typically seen as a test that evaluates the learning outcome, but its other functions may be ignored or undervalued. However, examinations could also be associated as tools that could activate and motivate students in their learning process and examinations that could offer a venue for a more creative learning process with taste of real life utilization.

\section{F. Major Findings}

This study introduces topics and facets, which are essential for enhancing student activity, creativity, and motivation at the NDU. Open book examination could also help to achieve better learning outcomes.

Action research approaches, including observations by lecturers, were compared to learning outcomes and feedback material to verify conclusions. In general, students thought that open book exams with take home arrangements offered more freedom, which was a good point. However, the intense workload was often pondered. The following findings were noticed in an action-based evaluation study:

1) Students found the open book test a meaningful and positive option in testing procedures.

2) The open book test was seen as laborious but students evaluated it as an efficient learning method.

3) If a test included alternative choices, students typically used more time in selecting the option on which to answer.

4) The timing was the most challenging task in home tests.

5) Time allocation also correlated with results. Those students who took more time also earned better grades.

6) The lecturer noticed that it is meaningful to divide the test to different parts (basic tasks and one main task) with given time constraints: 20 -hour window with a six-hour recommended time to perform. The students agreed with this conclusion. 
7) The most demanding task for the students in the test was making final conclusions and realizing that the length of the answer was not the main issue for final scoring.

\section{G. How to Enhance Learning with Examination Policy?}

One of the targets in the experimentation was that the remote, private hours and exams could provide space for learners' creativity. Can we activate students' deeper interests through a more versatile preparation for the exam and during the exam itself? On the other hand, how do we maintain a fair scoring policy in the test practice? Another challenge is to find and keep a proper level of difficulty in exams.

The purpose of exams is to get a versatile view of the students' competence level. A comprehensive set of questions and question types are needed to get a wide and often deep enough understanding of the matter. It is underlined that in educational situations, it is important to leave some responsibility to the students and cultivate their creative approaches and learner autonomy [12]. Thoroughly planned open book tests may be one step in this direction.

In normal lecture periods, students can ask questions. In an open book test that happens in normal classroom in NDU, there is some scope to ask questions. For example, questions can deal with the meaning of some assignment presented. In distance-type open book tests (home assignments), students also have had the ability to e-mail or directly discuss them by phone with the lecturer. Typically, "much significant learning is acquired through doing" [13]. In open book examinations, the question is also "about doing." This means that test situations may also serve learning aspects besides evaluative purposes. However, the test must always clearly indicate students' competences. When formal examinations are modified, it is necessary to evaluate whether new kinds of test situations favor a certain type of competence or offer a trustworthy view of the acquired knowledge level and know-how. Hence, the new approaches bring additional challenges.

\section{CONCLUSION}

Evaluation of the education process has a solid role in NDU's education strategy. Assessment is one of the key elements in learning processes because grading is based on examinations. It is essential to find out how information can be communicated and how learning outcomes can be tested in a way that is authentic, accurate, and justified. Open book examinations have received some positive feedback and results. Further development is necessary when trying to generate test models that could serve learning targets in a more versatile way. Future assessment tool should 1) serve as a test that clarifies learning outcome, 2) serve as an instrument that could motivate and courage students, 3) serve as an instrument that could activate and foster students' creativity potential, and 4) serve as an instrument that could complement the learning process. Continuous assessment is needed to find out new ways for more promising and interesting test situations.

\section{REFERENCES}

[1] E. Koshy, V. Koshy, and H. Waterman, Action Research in Healthcare, Sage, 2010.

[2] S. Kemmis and R. McTaggart, "Participatory action research," in Handbook of Qualitative Research, N. Denzin and Y. Lincoln Eds., Thousand Oaks, CA: Sage, 2000, pp. 567-605.

[3] Z. O'Leary, The Essential Guide to Doing Research, London: Sage, 2004.

[4] P. Race, S. Brown, and B. Smith, 500 Tips on Assessment, 2nd ed., London: Routledge, 2005.

[5] S. Brown and G. Wisker, Enabling Student Learning: Systems and Strategies, Routledge. 1996.

[6] S. Freeman, D. Haak, and M. Wenderoth, "Increased course structure improves performance in introductory biology," CBE - Life Sciences Education, vol. 10, pp. 175-186, Summer 2011.

[7] J. Tao and Z. Li, "A case study on computerized take-home testing: benefits and pitfalls," Int. Journal of Technology in Teaching and Learning, vol. 8, no. 1, pp. 33-43, 2012.

[8] M. McAlpine, Principles of Assessment, Luton, UK: The CAA Centre TLTP Project, HEFCE, 2002.

[9] L. Suskie, "Fair assessment practices: Giving students equitable opportunities to demonstrate learning," Fair Assessment Practices. AAHE Bulletin, May 2000.

[10] L. Rose, "Students as researchers: A framework for using action research principles to improve instruction," Int. Journal of Teaching and Learning in Higher Education, vol. 20 no. 2, pp. 284-291, 2009.

[11] C. Tang. (2003). Biggs' structure of the observed learning outcome. SOLO. [Online]. Available: http://www.tedi.uq.edu.au/downloads/Biggs_Solo.pdf

[12] P. Kahn, "Annotating mathematical material: A route to developing holistic understanding and learner autonomy," MSOR Connections, vol. 10 , no. 1, pp. 25-28, 2010.

[13] C. Rogers, Freedom to Learn: A View of What Education Might Become, 1st ed., Columbus, OH: Charles Merrill, 1969.

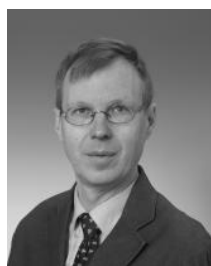

A. Rissanen was born in Helsinki. He obtained a licentiate in technology degree (electric engineering) in 1992 from the University of Technology in Tampere and his doctoral degree in physics in 2011 from the University of Helsinki in Finland. In 1997, he joined the National Defence University (Finland) as a senior lecturer in general technology. His research interests include science education, e-learning, new methods in PER. His previous research interest was in biomedical engineering. 